\title{
UNIQUELY ARCWISE CONNECTED PLANE CONTINUA HAVE THE FIXED-POINT PROPERTY
}

\author{
BY \\ CHARLES L. HAGOPIAN ${ }^{1}$
}

\begin{abstract}
This paper contains a solution to a fixed-point problem of G. S. Young [17, p. 884] and R. H. Bing [4, Question 4, p. 124]. Let $M$ be an arcwise connected plane continuum that does not contain a simple closed curve. We prove that every continuous function of $M$ into $M$ has a fixed point.
\end{abstract}

1. Introduction. A space $S$ has the fixed-point property if for each continuous function $f$ of $S$ into $S$, there exists a point $x$ of $S$ such that $f(x)=x$. It is known that every arcwise connected plane continuum that does not separate the plane has the fixed-point property [6], [7]. ${ }^{2}$ In this paper we consider another class of arcwise connected plane continua.

A continuum $M$ is uniquely arcwise connected if for each pair $p, q$ of points of $M$, there exists exactly one arc in $M$ with endpoints $p$ and $q$. Note that a continuum is uniquely arcwise connected if and only if it is arcwise connected and does not contain a simple closed curve. The sin $1 / x$ circle (Warsaw circle) is the simplest example of a uniquely arcwise connected plane continuum that separates the plane.

In [4, p. 123], Bing gave a dog-chases-rabbit argument that shows the $\sin 1 / x$ circle has the fixed-point property. Recently L. Mohler [12] used the Markov-Kakutani theorem (measure theory) to prove that every homeomorphism of a uniquely arcwise connected continuum into itself has a fixed point.

We prove that every uniquely arcwise connected plane continuum has the fixed-point property. An example of Young [17, p. 884] shows that this theorem cannot be extended to all uniquely arcwise connected continua in Euclidean 3-space.

Received by the editors October 4, 1977.

AMS (MOS) subject classifications (1970). Primary 54F20, 54F50, 54H25; Secondary 54C05, 55C20, 57A05.

Key words and phrases. Fixed-point property, uniquely arcwise connected continua, plane continua, indecomposable continua, Warsaw circle.

${ }^{1}$ The author presented this result at the Louisiana State University Topology Conference on March 11, 1977.

${ }^{2}$ It is not known whether every nonseparating plane continuum has the fixed-point property $[1$, p. 336], [4, Question 3, p. 122]. 
Our proof involves a continuous image of a ray defined by $\mathrm{K}$. Borsuk [5] and a nested sequence of polygonal disks constructed by K. Sieklucki [15]. In [5], Borsuk established the fixed-point property for every hereditarily unicoherent arcwise connected continuum. Sieklucki [15] and H. Bell [2] proved that every nonseparating plane continuum with a hereditarily decomposable boundary has the fixed-point property.

2. Preliminaries. A continuum is a nondegenerate compact connected metric space.

Throughout this paper $R^{2}$ is the Cartesian plane with metric $\rho$. We denote the boundary, closure, and interior of a given set $K$ by $\mathrm{Bd} K, \mathrm{Cl} K$, and Int $K$ respectively. The union of a collection $\mathcal{K}$ of sets is denoted by $\mathrm{St} \mathscr{K}$.

For each real number $\zeta$, let $I(\zeta)$ be the interval $\left\{(x, y) \in R^{2}: 0 \leqslant x \leqslant 1\right.$ and $y=\zeta\}$.

Definition. Suppose $A$ is an arc, $H$ is a continuum, and $A \cup H \subset R^{2}$. Then $H$ straddles $A$ if for each homeomorphism $h$ of $\operatorname{St}\{I(\zeta):-1 \leqslant \zeta \leqslant 1\}$ into $R^{2}$ with $h[I(0)]=A$, there exists a positive real number $\eta$ such that $H \cap h[I(\zeta)] \neq \varnothing$ when $|\zeta|<\eta$.

Henceforth $M$ is a uniquely arcwise connected continuum in $R^{2}$.

Notation. If $u$ and $v$ are distinct points of $M$, then the arc, the half-open arc, and the arc segment (open $\operatorname{arc}$ ) in $M$ with endpoints $u, v$ are denoted by $M[u, v], M[u, v)$, and $M(u, v)$ respectively.

Let $P$ be the image in $M$ of the ray $[1,+\infty)$ under a one-to-one continuous function $\psi$. For each positive integer $n$, let $a_{n}=\psi(n)$. The function $\psi$ determines a linear ordering $\prec$ of $P$ with $a_{1}$ as the first point. In this section, if $u$ and $v$ are points of $P$, the notation $M[u, v], M[u, v)$, and $M(u, v)$ will be used only when $u \prec v$.

Notation. For each point $u$ of $P$, let $P(u)$ denote $\{v \in P: u=v$ or $u \prec v\}$. Let $L=\bigcap_{n=1}^{\infty} \mathrm{Cl} P\left(a_{n}\right)$. In this section we assume $L$ is not degenerate. Hence $L$ is a continuum.

LEMMA 1. Suppose $u$ and $v$ are distinct points of $M$ that belong to $a$ complementary domain $D$ of $L$. Then $\mathrm{Cl} D$ contains $M[u, v]$.

Proof. Assume there is a point $w$ of $M(u, v)$ in $R^{2} \backslash \mathrm{Cl} D$. Let $J$ be an arc in $D$ that is irreducible between $M[u, w]$ and $M[w, v]$. Every arc in $M$ that intersects $D$ and $R^{2} \backslash \mathrm{Cl} D$ is straddled by $\mathrm{Bd} D$. Hence $\mathrm{Bd} D$ intersects each complementary domain of $J \cup M[u, v]$. Since $\operatorname{Bd} D \subset L$ and $M$ does not contain a simple closed curve, for each positive integer $n$, there is a point $z_{n}$ of $P\left(a_{n}\right)$ in $J$. Let $z$ be a limit point of $\left\{z_{n}\right\}_{n=1}^{\infty}$. It follows that $z \in J \cap L$, and this contradicts the fact that $J \subset R^{2} \backslash L$. Hence $M[u, v] \subset \mathrm{Cl} D$.

LEMMA 2. There exist a complementary domain $D$ of $L$ and a positive integer $n$ such that $\mathrm{Cl} D$ contains $P\left(a_{n}\right)$. 
Proof. Assume the contrary. Then by Lemma 1,

(1) for each complementary domain $D$ of $L$, there exists a positive integer $n$ such that $D \cap P\left(a_{n}\right)=\varnothing$.

Let $\left\{Y_{n}\right\}_{n=1}^{\infty}$ be the elements of a countable base for $M$ that intersect $L$. For each $n$, let $Z_{n}=\left\{z \in L \backslash\left\{a_{1}\right\}: Y_{n} \cap M\left[a_{1}, z\right]=\varnothing\right\}$.

Note that $L \backslash\left\{a_{1}\right\}=\cup_{n=1}^{\infty} Z_{n}$. To see this let $z$ be a point of $L \backslash\left\{a_{1}\right\}$. If $L \subset M\left[a_{1}, z\right]$, then $L$ is an arc and the closure of the complementary domain of $L$ (being $R^{2}$ ) contains $P$, contrary to our assumption. Thus $L \not \subset M\left[a_{1}, z\right]$. Hence there exists an integer $n$ such that $M\left[a_{1}, z\right] \subset M \backslash Y_{n}$. It follows that $z \in Z_{n}$. Therefore $L \backslash\left\{a_{1}\right\}=\cup_{n=1}^{\infty} Z_{n}$.

By the Baire category theorem, there exists an integer $\alpha$ such that $\mathrm{Cl} Z_{\alpha}$ contains a nonempty open subset $U$ of $L$. Let $Y$ and $Z$ be disjoint disks in $R^{2}$ such that

(2) $L \cap$ Int $Y \neq \varnothing$ and $L \cap$ Int $Z \neq \varnothing$ and

(3) $M \cap Y \subset Y_{\alpha}$ and $L \cap Z \subset U$.

Notation. For each component $C$ of $P \backslash Y$ that misses $a_{1}$, let Dom $C$ denote the complementary domain of $C \cup Y$ that misses $a_{1}$.

Let $\mathfrak{N}$ be the collection of all components $C$ of $P \backslash Y$ such that $a_{1} \notin C$ and $Z \cap \operatorname{Dom} C \neq \varnothing$. Note that each element of $\mathscr{N}$ is an arc segment with both endpoints in Bd $Y$.

For each element $C$ of $\Re$,

(4) $U \cap \operatorname{Dom} C=\varnothing$ and

(5) $C \cap$ Int $Z \neq \varnothing$.

Statement (4) is true; for otherwise, since $U \subset \mathrm{Cl} Z_{\alpha}, M \backslash Y_{\alpha}$ contains an arc $A$ that runs from $a_{1}$ to $U \cap \operatorname{Dom} C$, and $A \cup P$ contains a simple closed curve, violating the unique arcwise connectivity of $M$. Since $Z \cap$ Dom $C \neq \varnothing$ and $L \cap$ Int $Z$ is a nonempty subset of $U$, (5) follows immediately from (4).

Since $Y$ and $Z$ are disjoint, it follows from (2) that $\mathscr{T}$ is infinite. By (5), for each positive integer $n$,

(6) $P\left(a_{n}\right)$ contains all but finitely many elements of $\Re$.

For each element $C$ of $\Re$,

(7) Dom $C$ contains at most finitely many elements of $\mathscr{T}$ and

(8) only finitely many elements of $\Re$ separate $a_{1}$ from $C$ in $R^{2} \backslash Y$.

To verify (7) assume there exists an infinite subcollection $\Re$ of $\Re$ such that St $\Re \subset$ Dom $C$. By (5), each element of $\Re$ intersects $Z$. Hence by (6), there is a point $t$ of $L$ in $Z \cap \mathrm{Cl} \mathrm{St} \Re$. By (4), $t \notin$ Dom $C$. Therefore $t \in C$.

Let $J$ be an arc segment in Dom $C$ with endpoints in $C \backslash\{t\}$ such that the bounded complementary domain $K$ of $C \cup J$ has the following properties. The point $t$ belongs to $\mathrm{Cl} K$ and $U$ contains $K \cap L$.

Since infinitely many elements of $\mathcal{\vartheta}$ intersect $K$, it follows from (1) and (6) that no complementary domain of $L$ contains $K \cap \mathrm{St} \mathscr{\Re}$. Therefore $K \cap L$ $\neq \varnothing$. But since $K \subset$ Dom $C$, this contradicts (4). Hence (7) is true. 
To establish (8) assume the contrary. By (7), there exists a sequence $\left\{C_{n}\right\}_{n=1}^{\infty}$ of distinct elements of $\Re$ such that for each $n$, Dom $C_{n} \subset$ Dom $C_{n+1}$. By (1), (4), (5), (6), and Lemma 1, for some $n, L$ separates $C_{1}$ from $C_{n}$ in $Z$. Let $A$ be an arc segment in $Z$ such that $\mathrm{Cl} A$ is an arc irreducible between $C_{1}$ and $C_{n}$. Since $C_{1} \subset \operatorname{Dom} C_{n}, A \subset \operatorname{Dom} C_{n}$. But since $A \cap L$ $\neq \varnothing$, this contradicts (4). Hence (8) is true.

Let $\theta=\left\{C \in \Re\right.$ : no element of $\Re$ separates $a_{1}$ from $C$ in $\left.R^{2} \backslash Y\right\}$. Since $\mathfrak{T}$ is infinite, it follows from (7) and (8) that $\theta$ is infinite.

Next we define a pair $p, q$ of points of $P \cap \operatorname{Int} Y$. We consider two cases.

Case 1.1. Suppose $L \cap P \cap$ Int $Y \neq \varnothing$. Define $p$ to be a point of $L \cap P \cap$ Int $Y$, and let $q$ be a point of $(P(p) \cap$ Int $Y) \backslash\{p\}$.

Case 1.2. Suppose $L \cap P \cap$ Int $Y=\varnothing$. Applying (1), we define $p$ and $q$ to be points of $P \cap$ Int $Y$ such that $P(q)$ misses the $p$-component of $R^{2} \backslash L$.

An element $M(u, v)$ of $\theta$ in $P(q)$, a point $z$ of $M(u, v) \cap$ Int $Z$, and an arc segment $I$ in (Int $Z) \backslash \mathrm{Cl}$ Dom $M(u, v)$ exist such that

(9) $(\mathrm{Cl} I) \cap\left(M\left[a_{1}, v\right] \cup \operatorname{Bd} Z\right)=\{z\}$,

(10) $L \cap \mathrm{Cl} I \neq \varnothing$, and

(11) either $z \in L$ or $P \cap \mathrm{Cl} I=\{z\}$.

To verify this consider two cases.

Case 2.1. Suppose $L \cap P(q) \cap$ Int $Z \neq \varnothing$. Let $z$ be a point of $L \cap P(q) \cap$ Int $Z$. Let $M(u, v)$ be the element of $\Re$ that contains $z$. It follows from (4) that $M(u, v) \in \mathcal{O}$. Let $I$ be an arc segment in (Int $Z) \backslash \mathrm{Cl} \operatorname{Dom} M(u, v)$ that satisfies (9). Since $z \in L,(10)$ and (11) hold.

Case 2.2. Suppose $L \cap P(q) \cap$ Int $Z=\varnothing$. Note that $(L \backslash P) \cap$ Int $Z \neq \varnothing$. To see this assume otherwise. Since $L \cap \operatorname{Int} Z \neq \varnothing$ and $L \cap P(q) \cap \operatorname{Int} Z$ $=\varnothing$, there exists a point $a$ of $M\left[a_{1}, q\right]$ in $L \cap$ Int $Z$. Let $V$ be an open disk in $Z$ such that $a \in V$ and $V \backslash M\left[a_{1}, q\right]$ has exactly two components. Since $a \in L$, infinitely many elements of $\mathscr{N}$ intersect $V \backslash M\left[a_{1}, q\right]$. But since both components of $V \backslash M\left[a_{1}, q\right]$ are in $R^{2} \backslash L$, this contradicts (1) and (6).

Let $t$ be a point of $(L \backslash P) \cap$ Int $Z$. Let $W$ be an open disk in $Z$ that contains $t$ and misses $M\left[a_{1}, q\right]$. By (1), an element $M(w, x)$ of $\mathscr{R}$ and a point $r$ of $W \cap M(w, x)$ exist such that $W \cap P(x)$ misses the $r$-component $G$ of $R^{2} \backslash L$.

Let $T$ be an arc in $W$ that runs from $r$ to $t$. Define $s$ to be the first point of $T$ that belongs to $L$. Let $I$ be the arc segment in $T$ that precedes $s$ with the property that $\mathrm{Cl} I$ is irreducible between $s$ and $M[q, x]$. Define $z$ to be the endpoint of $I$ opposite $s$. Let $M(u, v)$ be the element of $\mathscr{N}$ that contains $z$. It follows from (4) that $s \notin \mathrm{Cl}$ Dom $M(u, v)$. Since $z \in G, P \cap \mathrm{Cl} I=\{z\}$. Hence by (4), $M(u, v) \in \mathcal{O}$. Clearly (9), (10), and (11) hold.

Let $J$ be a polygonal arc segment in

$$
Z \backslash\left(M\left[a_{1}, v\right] \cup \operatorname{Dom} M(u, v) \cup \mathrm{Cl} I\right)
$$


with endpoints $b$ and $b_{0}$ in $M(u, v)$ such that $z \in M\left(b, b_{0}\right) \subset Z$.

Define $K_{0}$ to be the component of $R^{2} \backslash\left(J \cup M\left[b, b_{0}\right]\right)$ that contains $I$. Note that $K_{0} \subset Z$.

The following statements and definitions $\left(12_{n}\right)-\left(21_{n}\right)$ will be used inductively.

By (7), (8), and (10), for $n=1$, there exists an element $M\left(w_{n}, x_{n}\right)$ of $\theta$ in $P(v)$ such that

$\left(12_{n}\right) K_{n-1} \cap M\left(w_{n}, x_{n}\right) \neq \varnothing$ and

$\left(13_{n}\right)$ no element of $\mathcal{O}$ in $M\left(b_{n-1}, w_{n}\right)$ intersects $K_{n-1}$.

For $n=1$,

$\left(14_{n}\right)$ let $H_{n}=M\left(b_{n-1}, x_{n}\right) \cap \mathrm{Cl} K_{n-1}$,

$\left(15_{n}\right)$ let $I_{n}$ be the component of $I \backslash M\left(p, x_{n}\right)$ whose closure contains $z$,

$\left(16_{n}\right)$ let $J_{n}$ be the arc in $\mathrm{Cl} J$ that is irreducible between $b$ and $M\left(w_{n}, x_{n}\right)$, and

$\left(17_{n}\right)$ let $b_{n}$ be the endpoint of $J_{n}$ that belongs to $M\left(w_{n}, x_{n}\right)$.

Let $J^{\prime}$ be the arc in $\mathrm{Cl} J$ that is irreducible between $b_{0}$ and $M\left(w_{1}, x_{1}\right)$. Since $Y \cup \operatorname{Dom} M(u, v)$ misses $M\left(w_{1}, x_{1}\right) \cup \mathrm{Cl} K_{0}$, there exists an arc segment $I^{\prime}$ in $Y \cup \operatorname{Dom} M(u, v)$ from $p$ to $z$ such that $\mathrm{Cl} I_{1}$ and $\mathrm{Cl} I^{\prime}$ abut on $M\left[b, b_{0}\right]$ from opposite sides with respect to the simple closed curve $\Gamma$ in $J_{1} \cup J^{\prime} \cup$ $M\left(b, b_{0}\right) \cup M\left(w_{1}, x_{1}\right)\left[13\right.$, Theorem 32, p. 181]. Since $\Gamma \cap\left(I_{1} \cup I^{\prime} \cup\{p\}\right)$ $=\varnothing, J_{1} \cup J^{\prime} \cup M\left(u, x_{1}\right)$ separates $p$ from $I_{1}$ in $R^{2}$. Since $J_{1} \cap J^{\prime}=\varnothing$, either $J_{1} \cup M\left(u, x_{1}\right)$ or $J^{\prime} \cup M\left(u, x_{1}\right)$ separates $p$ from $I_{1}$ in $R^{2}[13$, Theorem 20, p. 173]. For convenience we assume that $J_{1} \cup M\left(u, x_{1}\right)$ separates $p$ from $I_{1}$ in $R^{2}$.

For $n=1$,

$\left(18_{n}\right)$ let $K_{n}$ be the complementary domain of $J_{n} \cup M\left(b, b_{n}\right)$ that contains $I_{n}$.

Clearly, for $n=1$,

$\left(19_{n}\right) p \notin K_{n}$.

By (10) and (11), for $n=1$,

$\left(20_{n}\right) L \cap\left(K_{n} \cup\{z\}\right) \neq \varnothing$.

Next we show that for $n=1$,

$\left(21_{n}\right) K_{n} \cap Y \cap P\left(b_{n}\right)=\varnothing$.

Let $A$ be an arc in $J_{1}$ such that $A \cap M\left(p, x_{1}\right)=\{b\}$. Since $M(u, v)$ and $M\left(w_{1}, x_{1}\right)$ belong to $\Theta, M(u, v)$ and $M\left(w_{1}, x_{1}\right)$ are not separated in $R^{2} \backslash Y$ by an element of $\mathfrak{R}$. Hence there exists a polygonal arc segment $B$ in $R^{2} \backslash\left(Y \cup M\left(p, x_{1}\right)\right)$ such that $A \cup\left\{b_{1}\right\} \subset \mathrm{Cl} B$. Let $I_{1}^{\prime}$ be an arc in $\left(\mathrm{Cl} I_{1}\right) \backslash$ $B$ that contains $z$. Let $K$ be the complementary domain of $B \cup M\left[b, b_{1}\right]$ that intersects $I_{1}^{\prime}$.

Note that

(22) $K_{1} \cap Y \subset K$.

To see this let $y$ be a point of $K_{1} \cap Y$. We must show that $y \in K$. Let $F$ be a 
polygonal arc in $K_{1}$ from $y$ to $I_{1}^{\prime}$ such that $B \cap F$ is finite and $F$ crosses $B$ at each point of $B \cap F$. Clearly $y \in K$ if $B \cap F=\varnothing$, so we assume $B \cap F \neq \varnothing$. Since $I_{1}^{\prime} \cup Y \cup M(z, v)$ misses $B \cup J_{1}$, one complementary domain of $B \cup$ $J_{1}$ contains $I_{1}^{\prime} \cup\{y\}$. Since $F \cap J_{1}=\varnothing$, it follows that $F$ crosses $B$ an even number of times. Therefore $F$ crosses $B \cup M\left[b, b_{1}\right]$ an even number of times. Thus $y \in K$. Hence (22) is true.

It follows from (22) that $\left(21_{1}\right)$ can be established by proving

(23) $K \cap Y \cap P\left(b_{1}\right)=\varnothing$.

To verify (23) first note that since $p \notin K_{1}$ and $M[p, b) \cap\left(J_{1} \cup M\left(b, b_{1}\right)\right)$ $=\varnothing, I_{1}^{\prime}$ and $M[p, b]$ abut on $A \cup M[b, v]$ from opposite sides with respect to a simple closed curve in $J_{1} \cup M\left(b, b_{1}\right)$. Hence $I_{1}^{\prime}$ and $M[p, b]$ abut on $A \cup M[b, v]$ from opposite sides with respect to $B \cup M\left[b, b_{1}\right][13$, Theorem 32, p. 181]. Since $M[p, b) \cap\left(B \cup M\left[b, b_{1}\right]\right)=\varnothing$, it follows that $p \notin K$.

Let $Q$ be an arc from $p$ to $q$ in Int $Y$. Either $p \in L$ (Case 1.1) or $L \cap P \cap$ Int $Y=\varnothing$ and $P(q)$ misses the $p$-component of $R^{2} \backslash L$ (Case 1.2). Hence the $p$-component of $Q \backslash \mathrm{Cl} K$ contains a point $r$ of $L$. Let $G$ be an open set in $Y \backslash \mathrm{Cl} K$ that contains $r$.

Now suppose that (23) is false. Since $r \in L$, there exists an $\operatorname{arc} M[s, t]$ in $P\left(b_{1}\right)$ such that $s \in K \cap Y$ and $t \in G$.

Let $M\left(s^{\prime}, t^{\prime}\right)$ be an arc segment in $M[s, t] \backslash Y$ such that $s^{\prime} \in K \cap \operatorname{Bd} Y$ and $t^{\prime} \in(\operatorname{Bd} Y) \backslash K$. A component of $\left(B \cup M\left[b, b_{1}\right]\right) \backslash$ Int $Y$ separates $s^{\prime}$ from $t^{\prime}$ in $R^{2} \backslash$ Int $Y\left[13\right.$, Theorem 27, p. 177]. Since $B \cap M\left(b, b_{1}\right)=\varnothing$ and $M\left(s^{\prime}, t^{\prime}\right) \cap\left(Y \cup M\left(b, b_{1}\right)\right)=\varnothing$, it follows that $B \cup M[b, v] \cup M\left[w_{1}, b_{1}\right]$ separates $s^{\prime}$ from $t^{\prime}$ in $R^{2} \backslash$ Int $Y$. Hence $\left\{v, w_{1}\right\}$ separates $s^{\prime}$ from $t^{\prime}$ in $\mathrm{Bd} Y$. Thus $M\left(s^{\prime}, t^{\prime}\right)$ is an element of $\Re$ that separates $M(u, v)$ from $M\left(w_{1}, x_{1}\right)$ in $R^{2} \backslash Y[13$, Theorem 30, p. 158], and this contradicts the fact that $M(u, v)$ and $M\left(w_{1}, x_{1}\right)$ belong to $\theta$. Hence (23) is true. Consequently $\left(21_{1}\right)$ is true.

Proceeding inductively, for each integer $n>1$, we define $M\left(w_{n}, x_{n}\right), H_{n}, I_{n}$, $J_{n}, b_{n}$, and $K_{n}$ satisfying $\left(12_{n}\right)-\left(21_{n}\right)$. For $n>1,\left(12_{n}\right)$ and $\left(13_{n}\right)$ follow from (7), (8), and $\left(20_{n-1}\right)$. To verify $\left(19_{n}\right)$ for $n>1$, note that by $\left(19_{n-1}\right)$, there exists an $\operatorname{arc} A$ in $J_{n}$ such that $M[p, b]$ and $\mathrm{Cl} I_{n}$ abut on $A \cup M[b, v]$ from opposite sides with respect to a simple closed curve in $J_{n} \cup M\left(b, b_{n}\right)[13$, Theorem 32, p. 181]. The arguments given for $\left(20_{n}\right)$ and $\left(21_{n}\right)$ when $n=1$ hold when $n>1$.

Since $Y \cap Z=\varnothing$, for each positive integer $m$,

(24) there exists an integer $n$ such that $a_{m} \notin P\left(x_{n}\right)$.

Let $H$ be the limit superior of $\left\{H_{n}\right\}_{n=1}^{\infty}$. By (24), $H \subset L$.

Since $K_{0} \cap Y=\varnothing$, it follows from $\left(21_{n}\right)$ that

(25) $Y \cap \cup_{n=1}^{\infty} H_{n}=\varnothing$.

Since $\left\{J_{n}\right\}_{n=1}^{\infty}$ is a nested sequence of arcs, $\left\{b_{n}\right\}_{n=1}^{\infty}$ converges to a point $c$ of $H \cap J_{1}$. For each positive integer $n$, let $B_{n}$ be the polygonal arc in $J_{n}$ with endpoints $c$ and $b_{n}$. 
Since $J_{1} \cap M\left[a_{1}, u\right]=\varnothing$, it follows from $\left(13_{n}\right)$ and $\left(16_{n}\right)$ that $\left(J_{1} \backslash B_{1}\right) \cap$ $\cup_{n=1}^{\infty} H_{n}=\varnothing$. For each positive integer $n$, every component of $H_{n+1}$ intersects $B_{n}$. Hence $H$ is connected.

For each component $C$ of $P(u) \backslash Y$,

(26) $H \cap \operatorname{Int}(Y \cup \operatorname{Dom} C)=\varnothing$.

To see this let $x$ be the last point of $\mathrm{Cl} C$ with respect to the ordering of $P$. By $\left(13_{n}\right)$ and $\left(16_{n}\right), c \notin M(b, x)$. Let $i$ be a positive integer such that $B_{i} \cap$ $M(b, x)=\varnothing$. Since $c \in U$, it follows from (4) that $B_{i} \cap$ Dom $C=\varnothing$. Since $P$ does not contain a simple closed curve, $C \cap \cup_{n=i+1}^{\infty} H_{n}=\varnothing$. Since $B_{i}$ intersects each component of $\cup_{n=i+1}^{\infty} H_{n}$, it follows from (25) that $\cup_{n=i+1}^{\infty} H_{n}$ misses $Y \cup$ Dom $C$ [13, Theorem 28, p. 156]. Hence (26) is established.

Next we prove that Knaster's chainable indecomposable continuum with one endpoint [11, Example 1, p. 204] is a continuous image of $H$. We use a result [8, Theorem 1] that was derived from an argument of D. P. Bellamy [3]. According to Theorem 1 of [8], $H$ can be mapped continuously onto Knaster's continuum if there exists a sequence $\left\{G_{n}\right\}_{n=1}^{\infty}$ of nonempty open sets in $H$ such that $\left(\mathrm{Cl} G_{1}\right) \cap \mathrm{Cl} G_{2}=\varnothing$ and for each $n$,

$(27 n) G_{2 n+1} \cup G_{2 n+2} \subset G_{2 n-1}$ and

$\left(28_{n}\right)$ there exists a separation $E_{n} \cup F_{n}$ of $M \backslash G_{2 n}$ such that $G_{2 n+1} \subset E_{n}$ and $G_{2 n+2} \subset F_{n}$.

To establish the existence of $\left\{G_{n}\right\}_{n=1}^{\infty}$, order $B_{1}$ so that $b_{1}$ is its first point. Let $c_{1}$ be the first point of $B_{1} \cap L$ with respect to the ordering of $B_{1}$. By (1) and (24), $c_{1} \neq c$.

If $b_{1} \neq c_{1}$, define $C_{1}$ to be the arc in $B_{1}$ from $b_{1}$ to $c_{1}$. If $b_{1}=c_{1}$, let $C_{1}=\left\{c_{1}\right\}$.

Note that

(29) $c_{1} \in H$.

To see this consider two cases.

Case 3.1. Suppose $c_{1} \in P(z)$. Let $m$ be an integer such that $J_{m} \cap M\left[z, c_{1}\right]$ $=\varnothing$. For each integer $n \geqslant m, M\left[z, c_{1}\right] \subset \mathrm{Cl} K_{n}$. It follows from (7) that $c_{1} \in H$.

Case 3.2. Suppose $c_{1} \notin P(z)$. For some positive integer $n, C_{1} \cap P\left(a_{n}\right)=\varnothing$; for otherwise, by (1), $L \cap\left(C_{1} \backslash\left\{c_{1}\right\}\right) \neq \varnothing$, and this contradicts the definition of $c_{1}$. Let $d$ be the last point of $C_{1} \cap P$ that precedes $c_{1}$ with respect to the ordering of $B_{1}$. Since $C_{1} \cap M\left[a_{1}, z\right]=\varnothing, d \in P(z)$.

Let $\Delta$ be the arc in $C_{1}$ from $d$ to $c_{1}$. Let $M(w, x)$ be the $d$-component of $P \backslash Y$. Since $c_{1} \in U$ and $\Delta \cap P=\{d\}$, it follows from (4) that $M(w, x) \in \theta$.

Let $A$ be an arc in $J_{1} \backslash \Delta$ such that $A \cap M(p, x)=\{b\}$. Observe that

(30) $A$ and $\Delta$ abut on $M[u, x]$ from the same side.

To verify (30) first note that, by (4), $\Delta \cap \operatorname{Dom} M(w, x)=\varnothing$. Hence there exists a polygonal arc segment $B$ in $R^{2} \backslash(Y \cup M(p, x))$ such that $A \cup \Delta C$ $\mathrm{Cl} B$. Let $F$ be an arc in $R^{2} \backslash M(p, x)$ from $p$ to $x$ such that $B \cap F$ is finite 
and $F$ crosses $B$ at each point of $B \cap F$.

Suppose (30) is false. Then $F$ crosses $B$ an odd number of times. It follows that $p$ and $x$ are separated in $R^{2}$ by $B \cup M[b, d]$. By the argument for (23), there is a component of $P(z) \backslash Y$ that separates $M(u, v)$ from $M(w, x)$ in $R^{2} \backslash Y$, and this contradicts the fact that $M(u, v)$ and $M(w, x)$ belong to $\theta$. Hence (30) is true.

Let $i$ be a positive integer such that $J_{i} \cap M(b, x)=\varnothing$. Let $E$ be an arc in $\mathrm{Cl} I_{i}$ such that $E \cap M[u, x]=\{z\}$. Since $E$ and $A$ abut on $M[u, x]$ from the same side, it follows from (30) that $E$ and $\Delta$ abut on $M[b, x]$ from the same side. Since $\Delta \cap P=\{d\}$, for each integer $j \geqslant i, c_{1} \in K_{j}$.

Let $V$ be a disk in $K_{i}$ such that $c_{1} \in$ Int $V$. Since $c_{1} \in L, V \cap P\left(b_{i}\right) \neq \varnothing$. For each integer $j>i$, since $c_{1} \in K_{j}$, if $V \cap M\left(b_{i}, b_{j}\right)=\varnothing$, then $V \subset K_{j}$. Hence for some $j>i, H_{j}$ contains the first point of $V \cap P\left(b_{i}\right)$ with respect to the ordering of $P$ (recall $\left(14_{n}\right)$ ). It follows that $c_{1} \in H$. Thus (29) is established.

Let $D_{1}$ and $D_{2}$ be open disks in $R^{2}$ such that $B_{1} \subset D_{1}, \mathrm{Cl} I_{1} \subset D_{2}$, and $\left(\mathrm{Cl} D_{1}\right) \cap \mathrm{Cl} D_{2}=\varnothing$. Let $i_{1}=1$.

Let $j_{1}$ be an integer greater than 1 such that

(31) $C_{1} \cap P\left(w_{j_{1}}\right)=\varnothing$ and $J_{j_{1}} \cap M\left[z, b_{1}\right]=\varnothing$.

By (7), (8), (10), and (11), there exists an integer $i_{2}>j_{1}$ such that $D_{2} \cap K_{1} \cap$ $M\left(w_{i_{2}}, x_{i_{2}}\right) \neq \varnothing$.

Let $\Lambda$ be an arc segment in

$\left((\mathrm{Bd} Y) \cap \mathrm{Cl} \operatorname{Dom} M\left(w_{i_{2}}, x_{i_{2}}\right)\right) \backslash M\left[p, x_{i_{2}}\right]$

that has $x_{i_{2}}$ as an endpoint. Let $\Lambda_{1}$ be a polygonal arc segment in Dom $M\left(w_{i_{2}}, x_{i_{2}}\right) \backslash M\left[b, b_{i}\right]$ from a point $e$ of $\Lambda$ to $D_{2} \cap K_{1}$ such that $B_{1} \cap \Lambda_{1}$ is finite and $\Lambda_{1}$ crosses $B_{1}$ at each point of $B_{1} \cap \Lambda_{1}$.

By $\left(21_{1}\right), e \notin K_{1}$.

Let $\Pi_{1}$ be the arc in $B_{1}$ from $c_{1}$ to $c$. Since $\Lambda_{1}$ misses $M\left[b, b_{1}\right] \cup\left(J_{1} \backslash \Pi_{1}\right)$, it follows that $\Lambda_{1}$ crosses $\Pi_{1}$ an odd number of times.

By (26), there exists a simple closed curve $\Sigma_{1}$ in

$$
Y \cup \Lambda_{1} \cup D_{2} \cup \operatorname{Dom} M(u, v)
$$

such that $\Lambda_{1} \subset \Sigma_{1}, H \cap \Sigma_{1} \subset D_{2}$, and $\Pi_{1} \cap \Sigma_{1} \subset \Lambda_{1}$. Since $\Pi_{1}$ crosses $\Sigma_{1}$ an odd number of times, $\Sigma_{1}$ separates $c$ from $c_{1}$ in $R^{2}$.

Define $\Omega_{1}$ to be the $c$-component of $R^{2} \backslash \Sigma_{1}$. Note that $B_{i_{2}} \subset \Omega_{1} \subset R^{2} \backslash C_{1}$.

Let $E_{1}=\Omega_{1} \cap\left(H \backslash D_{2}\right)$ and $F_{1}=H \backslash\left(D_{2} \cup E_{1}\right)$.

For $i=1$ and 2, let $G_{i}=D_{i} \cap H$. Note that $E_{1} \cup F_{1}$ is a separation of $H \backslash G_{2}$.

Let $c_{2}$ be the first point of $L \cap B_{i_{2}}$ with respect to the ordering of $B_{1}$. By (1) and (24), $c_{2} \neq c$.

If $b_{i_{2}} \neq c_{2}$, define $C_{2}$ to be the arc in $B_{1}$ from $b_{i_{2}}$ to $c_{2}$. If $b_{i_{2}}=c_{2}$, let $C_{2}=\left\{c_{2}\right\}$. By the argument for (29), $c_{2} \in H$. 
Let $D_{3}$ and $D_{4}$ be open disks such that $B_{i_{2}} \subset D_{3} \subset D_{1} \cap \Omega_{1}$ and $C_{1} \subset D_{4}$ $\subset D_{1} \backslash \Omega_{1}$. Note that $D_{3} \cap H \subset E_{1}$ and $D_{4} \cap H \subset F_{1}$.

It follows from (31) and the arguments for Cases 3.1 and 3.2 that $c_{1} \in \mathrm{Cl} K_{i}$ for each integer $i \geqslant i_{2}$.

Proceeding inductively, we let $n$ be an integer greater than 1 . We assume that for each integer $m(1<m \leqslant n)$, an integer $i_{m}$, a point $c_{m}$ of $H \backslash\{c\}$, a subset $C_{m}$ of $B_{1}$, and disjoint open disks $D_{2 m-1}, D_{2 m}$ have been defined such that

$\left(32_{m}\right) c_{m}$ is the first point of $L \cap B_{i_{m}}$ with respect to the ordering of $B_{1}$,

$\left(33_{m}\right) C_{m}$ is a minimal connected set containing $\left\{b_{i_{m}}, c_{m}\right\}$,

$\left(34_{m}\right) B_{i_{m}} \subset D_{2 m-1}$,

$\left(35_{m}\right) C_{m-1} \subset D_{2 m}$, and

$\left(36_{m}\right) c_{m-1} \in \mathrm{Cl} K_{i}$ for each integer $i \geqslant i_{m}$.

For each integer $i(2<i \leqslant 2 n)$, let $G_{i}=D_{i} \cap H$. We assume that for each positive integer $i$ less than $n,\left(27_{i}\right)$ and $\left(28_{i}\right)$ are satisfied.

Let $j_{n}$ be an integer greater than $i_{n}$ such that $C_{n} \cap P\left(w_{j_{n}}\right)=\varnothing$ and $J_{j_{n}} \cap$ $M\left[z, b_{i_{n}}\right]=\varnothing$. Define $i_{n+1}$ to be an integer greater than $j_{n}$ such that

$$
D_{2 n} \cap K_{i_{n}} \cap M\left(w_{i_{n+1}}, x_{i_{n+1}}\right) \neq \varnothing .
$$

Let $\Pi_{n}$ be the arc in $B_{1}$ from $c_{n}$ to $c$. Define $\Lambda_{n}$ to be a polygonal arc segment in Dom $M\left(w_{i_{n+1}}, x_{i_{n+1}}\right)$ from (Bd $\left.Y\right) \backslash H$ to $D_{2 n} \cap K_{i_{n}}$ that crosses $\Pi_{n}$ an odd number of times.

Let $\Sigma_{n}$ be a simple closed curve in

$$
Y \cup \Lambda_{n} \cup D_{2 n} \cup \operatorname{Dom} M\left(w_{i_{n-1}}, x_{i_{n-1}}\right)
$$

such that $\Lambda_{n} \subset \Sigma_{n}, H \cap \Sigma_{n} \subset D_{2 n}$, and $\Pi_{n} \cap \Sigma_{n} \subset \Lambda_{n}$. Since $\Pi_{n}$ crosses $\Sigma_{n}$ an odd number of times, $\Sigma_{n}$ separates $c$ from $c_{n}$ in $R^{2}$.

Define $\Omega_{n}$ to be the $c$-component of $R^{2} \backslash \Sigma_{n}$. Let $E_{n}=\Omega_{n} \cap\left(H \backslash D_{2 n}\right)$ and $F_{n}=H \backslash\left(D_{2 n} \cup E_{n}\right)$.

To complete the inductive step define $c_{n+1}, C_{n+1}, D_{2 n+1}, D_{2 n+2}$ satisfying $\left(32_{n+1}\right)-\left(36_{n+1}\right),\left(27_{n}\right)$, and $\left(28_{n}\right)$ when $G_{2 n+1}=D_{2 n+1} \cap H$ and $G_{2 n+2}=$ $D_{2 n+2} \cap H$. It follows from the existence of $\left\{G_{n}\right\}_{n=1}^{\infty}$ that Knaster's continuum is a continuous image of $H$ [8, Theorem 1].

Since Knaster's continuum is indecomposable, $H$ contains an indecomposable continuum $\Phi[11$, Theorem 4, p. 208]. According to a theorem of $J$. Krasinkiewicz [10, Theorem 3.1], $\Phi$ has a composant $\Psi$ with the property that

(37) no arc segment in $R^{2} \backslash \Psi$ has an endpoint in $\Psi$.

Note that

(38) $\Psi \cap P(u)=\varnothing$.

To see this assume the contrary. Let $t$ be a point of $\Psi \cap P(u)$. Since $\Psi \subset H \subset M \backslash$ Int $Y$, it follows from (37) that $t \notin Y$. Let $C$ be the $t$ component of $P(u) \backslash Y$. By (37), $\Psi \cap \operatorname{Dom} C \neq \varnothing$, and this contradicts (26). Hence (38) is true. 
Since $M$ is arcwise connected, there exists an $\operatorname{arc} A$ in $M$ that intersects both $\Psi$ and $M \backslash \Psi$. By (37), there exist points $r$ and $s$ of $A \cap \Psi$ such that $M(r, s) \not \subset \Psi$. Let $B$ be a continuum in $\Psi$ that contains $\{r, s\}$. Note that $A \cup B$ separates $R^{2}$ [13, Theorem 22, p. 175]. By (37), each component of $R^{2} \backslash(A \cup B)$ intersects $\Psi$. Since $\Psi \subset L$, it follows from (38) that $A \cup P$ contains a simple closed curve, and this violates the unique arcwise connectivity of $M$. This contradiction completes the proof of Lemma 2.

LEMMA 3. For each positive integer $n, P\left(a_{n}\right)$ intersects $M \backslash L$.

Proof. Suppose $P\left(a_{n}\right) \subset L$ for some positive integer $n$. Assume without loss of generality that $P \subset L$. It follows from the proof of Lemma 2 (with Cases 1.2, 2.2, and 3.2 deleted) that this assumption involves a contradiction.

We assume without loss of generality that $D$ (in Lemma 2 ) is the unbounded complementary domain of $L$ and $P \subset \mathrm{Cl} D$. We also assume without loss of generality that $a_{1} \in D$ (Lemma 3 ).

Let $L^{\prime}$ be a subcontinuum of $L$. Define $D^{\prime}$ to be the unbounded complementary domain of $L^{\prime}$. Note that $D \subset D^{\prime}$.

Let $X$ be the nonseparating plane continuum $R^{2} \backslash D^{\prime}$. Since $M$ is arcwise connected, there is an arc segment $S$ in $M \cap D^{\prime}$ with an endpoint $s$ in $X$. Since $M$ does not contain a simple closed curve, we can assume without loss of generality that $P \cap \mathrm{Cl} S=\varnothing$.

Sieklucki [15, Lemma 5.5, p. 267] proved that $X$ has the following properties. There exists a sequence $\left\{Q_{n}\right\}_{n=1}^{\infty}$ of disks in $R^{2}$ such that $X=$ $\cap_{n=1}^{\infty} Q_{n}$ and for each $n$,

(i) $Q_{n+1} \subset$ Int $Q_{n}$,

(ii) the boundary $B_{n}$ of $Q_{n}$ is a polygonal simple closed curve with consecutive vertices $b_{n}^{1}, b_{n}^{2}, \ldots, b_{n}^{\mu_{n}}, b_{n}^{\mu_{n}+1}=b_{n}^{1}$, and

(iii) for $j=1,2, \ldots, \mu_{n}$, the interval in $B_{n}$ from $b_{n}^{j}$ to $b_{n}^{j+1}$ has diameter less than $2^{-n}$.

For every $b_{n}^{j}\left(n=1,2, \ldots\right.$ and $\left.j=1,2, \ldots, \mu_{n}\right)$ there exists a vertex $b_{n+1}^{\nu(j)}$ such that

(iv) the interval $N_{n}^{j}$ in $R^{2}$ from $b_{n}^{j}$ to $b_{n+1}^{\nu(j)}$ has diameter less than $2^{-n}$,

(v) $N_{n}^{j} \backslash\left\{b_{n}^{j}, b_{n+1}^{\nu(j)}\right\} \subset$ (Int $\left.Q_{n}\right) \backslash Q_{n+1}$, and

(vi) $N_{n}^{j} \cap N_{n}^{k}=\varnothing$ for each integer $k \neq j\left(1 \leqslant k \leqslant \mu_{n}\right)$.

Let $N=\cup_{n=1}^{\infty} \cup{ }_{j=1}^{p_{n}} N_{n}^{j}$. Note that each component of $N$ is a half-open $\operatorname{arc}$ in $R^{2} \backslash X$ with an endpoint in $X$.

Let $m$ be a given positive integer. Define $N_{m}$ to be the union of all components of $N$ that intersect $B_{m}$. Let $O_{m}$ be a subset of $N_{m}$ that is maximal with respect to the property that each component of $O_{m}$ is a component of $N_{m}$ and each pair of components of $O_{m}$ with a common endpoint is separated in $Q_{m} \backslash X$ by another pair of components of $O_{m}$. 
Let $c_{m}^{1}, c_{m}^{2}, \ldots, c_{m}^{\xi_{m}}, c_{m}^{\xi_{m}+1}=c_{m}^{1}$ denote the consecutive vertices of $B_{m}$ that belong to $O_{m}$. Since $X$ is not degenerate, we can assume without loss of generality that $\xi_{m}>3$. Assume without loss of generality that $B_{m} \cap S \neq \varnothing$.

Let $n$ be an integer greater than $m$. Define $E$ to be the closure of a component of $Q_{n} \backslash\left(O_{m} \cup X\right)$. We call $E$ an $(m, n)$-link on $X$. The polygonal arc $B_{n} \cap E$ is called the bottom of $E$. The two components of $E \cap O_{m}$ are called the sides of $E$. Note that the sides of $E$ are half-open $\operatorname{arcs}$ in $Q_{n} \backslash X$ with distinct endpoints in $X$. The diameter of the union of the sides of $E$ is less than $2^{3-m}$.

For $j=1,2, \ldots, \xi_{m}$, let $E_{j}$ be the $(m, n)$-link whose sides are contained in the components of $O_{m}$ that intersect $\left\{c_{m}^{j}, c_{m}^{j+1}\right\}$.

Suppose there exist two $(m, n)$-links $E$ and $F$ that have a common side such that $Q_{n} \cap S \subset E \cup F$ and $\mathrm{Cl} S$ misses the closure of each uncommon side of $E$ and $F$. Change the indexing of the ( $m, n)$-links (if necessary) so that $E=E_{1}, F=E_{\xi_{m}}$, and each pair of consecutive links has a common side.

Define $F_{1}$ to be the closure of the component of $\left(E_{1} \cup E_{\xi_{m}}\right) \backslash(S \cup X)$ that contains a side of $E_{2}$. Let $F_{j}=E_{j}$ for $1<j<\xi_{m}$. Define $F_{\xi_{m}}$ to be the closure of the component of $\left(E_{1} \cup E_{\xi_{m}}\right) \backslash(S \cup X)$ that contains a side of $E_{\xi_{m}-1}$. We call $\mathscr{F}=\left\{F_{j}: 1 \leqslant j \leqslant \xi_{m}\right\}$ an $m$-chain on $(X, S)$. We call $F_{1}$ and $F_{\xi_{m}}$ the end links of $\mathscr{F}$. Each $F_{j}\left(1<j<\xi_{m}\right)$ is called an interior link of $\mathscr{F}$. Let $T$ be the arc in $\mathrm{Cl} S$ that is irreducible between $s$ and $B_{n}$. The half-open arc $T \backslash\{s\}$ is called the common side of $F_{1}$ and $F_{\xi_{m}}$.

Since $S$ is an arc segment, for each positive integer $m$, there exists an $m$-chain on $(X, S)$.

Lemma 4. For each positive integer $i$, there exists an m-chain $\mathcal{F}$ on $(X, S)$ such that $m>i$ and no pair of consecutive links of $\mathscr{F}$ contains $\mathrm{Bd} X$ in its union.

Proof. Let $m$ and $m^{\prime}$ be integers such that $0<m \leqslant m^{\prime}$. Suppose $\mathscr{F}$ is an $m$-chain on $(X, S)$ and $U$ is the union of a pair of consecutive links of an $m^{\prime}$-chain on $(X, S)$. Then the union of some pair of consecutive links of $\mathscr{F}$ contains $U \cap \mathrm{Bd} X$. Hence it is sufficient to show that there exists an $m$-chain $\mathscr{F}$ on $(X, S)$ such that no pair of consecutive links of $\mathscr{F}$ contains Bd $X$ in its union.

Assume that for each positive integer $m$, every $m$-chain on $(X, S)$ has a pair of consecutive links whose union contains $\mathrm{Bd} X$. Then for each $m$, there exist a positive number $\varepsilon_{m}$, a pair of consecutive links $E_{m}, F_{m}$ of an $m$-chain on $(X, S)$, and an arc segment $A_{m}$ in $B_{m} \cup O_{m} \cup S$ such that $\left\{\varepsilon_{m}\right\}_{m=1}^{\infty}$ converges to zero, $\mathrm{Bd} X \subset E_{m} \cup F_{m}, A_{m}$ has diameter less than $\varepsilon_{m}$ and contains the uncommon sides of $E_{m}$ and $F_{m}$, and $A_{m} \cup X$ separates (Int $E_{m}$ ) $\backslash X$ from $R^{2} \backslash Q_{m}$ in $R^{2}$.

For each positive integer $m$, let $x_{m}$ and $y_{m}$ be the endpoints of $A_{m}$. Note 
that for each $m,\left\{x_{m}, y_{m}\right\} \subset \operatorname{Bd} X$. For each $m$, let $W_{m}$ be the complementary domain of $A_{m} \cup X$ whose closure contains $E_{m} \cup F_{m}$. Let $y$ be a limit point of $\left\{y_{m}\right\}_{m=1}^{\infty}$.

The continuum $\mathrm{Bd} X$ is nonaposyndetic at $y$ with respect to each point of $(\mathrm{Bd} X) \backslash\{y\}$ [9]. For assume otherwise. Then a continuum $Y$, an open disk $G$, and a point $z$ of $(\mathrm{Bd} X) \backslash \mathrm{Cl} G$ exist such that

$$
y \in G \cap \operatorname{Bd} X \subset Y \subset(\operatorname{Bd} X) \backslash\{z\} .
$$

Let $Z$ be an open disk such that $z \in Z \subset R^{2} \backslash(G \cup Y)$.

Let $i$ be an integer such that $B_{i} \cap Z \neq \varnothing$. Define $m$ to be an integer greater than $i$ such that $A_{m} \subset G$. Let $p$ be a point of $Z \cap\left(Q_{i} \backslash Q_{m}\right)$. Let $q$ be a point of $W_{m} \cap Z$.

There exists a polygonal arc $I$ in $Q_{i} \backslash X$ from $p$ to $q$ such that $A_{m} \cap I$ is finite and $I$ crosses $A_{m}$ at each point of $A_{m} \cap I$. Since $A_{m}$ separates $p$ from $q$ in $Q_{i} \backslash X, I$ crosses $A_{m}$ an odd number of times. It follows that $I \cup Z$ contains a simple closed curve that separates $x_{m}$ from $y_{m}$ in $R^{2}$. Since $\left\{x_{m}, y_{m}\right\} \subset Y \subset R^{2} \backslash(I \cup Z)$, this violates the connectivity of $Y$. Hence $\operatorname{Bd} X$ is nonaposyndetic at $y$ with respect to each point of $(\operatorname{Bd} X) \backslash\{y\}$.

According to a theorem of H. E. Schlais [14, Theorem 9], [8, Theorem 4], Bd $X$ contains an indecomposable continuum $\Phi$. Let $\Psi$ be a composant of $\Phi$ with the property that no arc segment in $R^{2} \backslash \Psi$ has an endpoint in $\Psi$ [10, Theorem 3.1].

Note that $\Psi \cap P=\varnothing$. To see this assume there is a point $u$ of $P$ in $\Psi$. By Lemma 3, there is a point $v$ of $P(u)$ in $D$. Let $J$ be an arc in $D$ that is irreducible between $M\left[a_{1}, u\right]$ and $M[u, v]$. Since $M\left[a_{1}, v\right) \cap P(v)=\varnothing$ and each complementary domain of $J \cup M\left[a_{1}, v\right]$ intersects $\Psi$, for each positive integer $n, J \cap P\left(a_{n}\right) \neq \varnothing$. Thus $J \cap L \neq \varnothing$, and this contradicts the definition of $J$. Hence $\Psi \cap P=\varnothing$.

From the last paragraph in the proof of Lemma 2, we see that the existence of $\Psi$ implies that $M$ contains a simple closed curve. This contradiction completes the proof of Lemma 4.

LemMA 5. Suppose $F$ is an element of an m-chain $\mathscr{F}, u$ is a point of $(P \cap \operatorname{Int} F) \backslash X, v$ is a point of $P \backslash F$, and $\mathrm{St} \mathscr{F}$ contains $M[u, v]$. Then $M[u, v]$ intersects the closure of a side of $F$.

Proof. Assume $M[u, v]$ misses the closure of each side of $F$. By Lemma 3, there is a point $y$ of $P(v)$ in $D$. Let $x$ be a point of $X \cap M[u, v] \cap \mathrm{Bd} F$ such that every arc in $M[u, x]$ from $P \backslash X$ to $x$ intersects Int $F$, and every arc in $M[x, y]$ from $x$ to $P \backslash X$ intersects $P \backslash F$. Let $J$ be an arc in $D$ that is irreducible between $M\left[a_{1}, x\right]$ and $M[x, y]$.

The continuum $X \cap \operatorname{Bd} F$ straddles every arc in $P$ that contains $x$ and has both endpoints in $D$. Consequently each complementary domain of $J \cup$ $M\left[a_{1}, y\right]$ intersects $X \cap \operatorname{Bd} F$. Since $M\left[a_{1}, y\right) \cap P(y)=\varnothing$, it follows that 
$J \cap L \neq \varnothing$, and this contradicts the definition of $J$. Hence $M[u, v]$ intersects the closure of a side of $F$.

In the remaining part of this section we assume $L=L^{\prime}$. Hence $D=D^{\prime}$ and $L$ is the boundary of the nonseparating plane continuum $X$.

Let $\mathscr{F}=\left\{F_{j}: 1 \leqslant j \leqslant \xi_{m}\right\}$ be an $m$-chain on $(X, S)$. Let $\mathcal{G}$ be the set of all elements $F_{j}$ of $\mathscr{F}$ such that for each point $u$ of $P, P(u)$ intersects (Int $\left.F_{j}\right) \backslash X$. It follows from Lemma 3 that $\mathcal{G}$ is not empty. Let $F_{i}$ and $F_{k}$ be the first and last links, respectively, of $\mathscr{F}$ that belong to $\mathcal{G}$.

Lemma 6. Suppose $B$ is an arc segment in $F_{j} \backslash X(i<j<k)$ that has an endpoint in $X$. Then there exists a point $u$ of $P$ such that each arc in $P(u)$ that intersects both (Int $\left.F_{i}\right) \backslash X$ and (Int $\left.F_{k}\right) \backslash X$ also intersects $B$.

Proof. Let $\mathcal{H}$ be an $m$-chain on $(X, S)$ such that $B$ intersects the bottom of a link of $\mathcal{H}$. Define $u$ to be a point of $P$ such that $P(u) \subset(\mathrm{St} \mathcal{H}) \backslash((\mathrm{Cl} B)$ $\backslash B)$.

Suppose there is an $\operatorname{arc} M[v, w]$ in $P(u) \backslash B$ that intersects both (Int $\left.F_{i}\right) \backslash X$ and (Int $\left.F_{k}\right) \backslash X$. Assume without loss of generality that $v \in\left(\operatorname{Int} F_{i}\right) \backslash X$ and $w \in\left(\right.$ Int $\left.F_{k}\right) \backslash X$.

Let $V$ be the $v$-component of $(S t \mathcal{H}) \backslash(B \cup S \cup X)$. Note that $V$ misses $F_{k}$ and contains $(\mathrm{St} \mathcal{H}) \cap\left(\left(\operatorname{Int} F_{i}\right) \backslash X\right)$. Since $M[v, w] \cap \mathrm{Cl}(B \cup S)=\varnothing$, the continuum $X \cap \mathrm{Bd} V$ straddles each subarc of $M[v, w]$ that has one endpoint in $V$ and the other endpoint in $M \backslash \mathrm{Cl} V$.

Since $F_{i} \in \mathcal{G}$, there is a point $z$ of $P(w)$ in (Int $\left.F_{i}\right) \backslash X$. Let $J$ be an arc in $V$ that is irreducible between $M[v, w]$ and $M[w, z]$. Each complementary domain of $J \cup M[v, z]$ intersects $X \cap \operatorname{Bd} V$. Since $M[v, z) \cap P(z)=\varnothing$, it follows that $J \cap L \neq \varnothing$, and this contradicts the definition of $J$. Hence each arc in $P(u)$ that intersects (Int $\left.F_{i}\right) \backslash X$ and (Int $\left.F_{k}\right) \backslash X$ intersects $B$.

It follows from Lemma 6 that $\mathcal{G}$ is the subchain $\left\{F_{j}: i \leqslant j \leqslant k\right\}$ of $\mathscr{F}$.

Definition. Suppose $K$ is an arcwise connected subset of $M$ that is contained in (St $\mathcal{G}) \backslash \mathrm{Bd}(X \cup \mathrm{St} \mathscr{F})$ and intersects Int $F_{i}$ and Int $F_{k}$. Then $K$ is a trace of $\mathcal{G}$ if for each arc $A$ in $K$, there exists a function $g$ of $A$ into $\mathcal{G}$ with the following properties:

(1) For each point $a$ of $A, a \in g(a)$.

(2) If $a$ and $b$ are points of $A$ and $g(a) \neq g(b)$, then $M[a, b]$ intersects a side of $g(a)$ and the interior of each link of $\mathcal{G}$ between $g(a)$ and $g(b)$ (with respect to the index ordering of $\mathcal{G}$ ).

Definition. An arcwise connected set $K$ agrees with $\mathcal{G}$ if $K$ is a trace of $\mathcal{G}$, $\mathcal{G} \backslash\left\{F_{i}\right\}, \mathcal{G} \backslash\left\{F_{k}\right\}$, or $\mathcal{G} \backslash\left\{F_{i}, F_{k}\right\}$.

Lemma 7. There exists a point $u$ of $P \cap \operatorname{Int} F_{i}$ such that $P(u)$ is a trace of $\mathcal{G}$.

Proof. Let $W=\{x \in X: x$ is an endpoint of a side of a link of $\mathcal{G}\}$. Define $u$ to be a point of $P \cap\left(\left(\operatorname{Int} F_{i}\right) \backslash X\right)$ such that $P(u)$ is contained in St $\mathscr{F}$ and 
misses $W \cup \operatorname{Bd}(X \cup \operatorname{St} \mathscr{F})$ and $\cup\left\{\left(\operatorname{Int} F_{j}\right) \backslash X: 1 \leqslant j \leqslant i\right.$ or $\left.k<j \leqslant \xi_{m}\right\}$.

Using Lemma 5 , we define a function $g^{*}$ of $P(u)$ onto $\mathcal{G}$ such that

(i) $v \in g^{*}(v)$ for each point $v$ of $P(u)$ and

(ii) if $v$ and $w$ are points of $P(u)$ and $g^{*}(v) \neq g^{*}(w)$, then $M[v, w]$ intersects a side of $g^{*}(v)$ and the interior of each link of $\mathcal{G}$ between $g^{*}(v)$ and $g^{*}(w)$.

By considering the restriction of $g^{*}$ to each arc in $P(u)$, we see that $P(u)$ is a trace of $\mathcal{G}$.

\section{Principal result.}

THEOREM. If $M$ is a uniquely arcwise connected plane continuum, then $M$ has the fixed-point property.

Proof. Assume there exists a continuous function $f$ of $M$ into $M$ that moves each point of $M$. Let $\varepsilon$ be a positive number such that

(1) $\rho(z, f(z))>\varepsilon$ for each point $z$ of $M$.

According to Borsuk [5], there exists a sequence $\left\{a_{n}\right\}_{n=1}^{\infty}$ of points of $M$ such that for each $n$,

(2) $\rho\left(a_{n}, a_{n+1}\right)=\varepsilon / 3\left[5\right.$, p. $\left.19,\left(4_{n}\right)\right]$,

(3) if $z \in M\left(a_{n}, a_{n+1}\right)$, then $\rho\left(a_{n}, z\right)<\varepsilon / 3\left[5\right.$, p. $\left.19,\left(5_{n}\right)\right]$,

(4) $M\left[a_{1}, a_{n}\right] \cap M\left[a_{n}, a_{n+1}\right]=\left\{a_{n}\right\}$ for $n>1[5$, p. 19, (11)], and

(5) $\left\{a_{n}, a_{n+1}\right\} \subset M\left[a_{1}, f\left(a_{n}\right)\right)\left[5\right.$, p. $\left.19,\left(7_{n}\right),(13)\right]$.

For each positive integer $n$, let $\psi_{n}$ be a homeomorphism of the half-open real line interval $[n, n+1)$ onto $M\left[a_{n}, a_{n+1}\right)$. For each point $x$ of $[1,+\infty)$, let $\psi(x)=\psi_{n}(x)$ if $n \leqslant x<n+1$.

Let $P=\cup_{n=2}^{\infty} M\left[a_{1}, a_{n}\right)$. It follows from (4) that $\psi$ is a one-to-one continuous function of $[1,+\infty)$ onto $P$.

The function $\psi$ determines a linear ordering $\prec$ of $P$ with $a_{1}$ as the first point. As in $\$ 2$, for each point $u$ of $P$, we let $P(u)$ denote $\{v \in P: u=v$ or $u \prec v\}$.

For each point $u$ of $P$,

(6) $u \in M\left[a_{1}, f(u)\right]$.

To see this assume $u \notin M\left[a_{1}, f(u)\right]$. Suppose $u \in M\left[a_{n}, a_{n+1}\right)$. Since $M$ does not contain a simple closed curve, $a_{n+1} \notin M\left[a_{1}, f(u)\right]$. By (1), (2), and (3), $a_{n+1} \notin f\left[M\left[u, a_{n+1}\right]\right]$. Thus $M\left[a_{1}, f(u)\right] \cup f\left[M\left[u, a_{n+1}\right]\right]$ misses $a_{n+1}$ and contains an arc that runs from $a_{1}$ to $f\left(a_{n+1}\right)$, and this contradicts (5). Hence (6) holds.

By (2), $P \not \subset M\left[a_{1}, f\left(a_{1}\right)\right]$. Since $M$ does not contain a simple closed curve, there exists a point $a$ of $P$ such that $P(a) \cap M\left[a_{1}, f\left(a_{1}\right)\right]=\{a\}$.

Next we prove that

(7) $a \in f\left[M\left[a_{1}, a\right)\right]$.

Statement (7) is obviously true if $f\left(a_{1}\right)=a$, so we assume $f\left(a_{1}\right) \neq a$. Suppose $a \in M\left[a_{n}, a_{n+1}\right)$. By (5), $a_{2} \in M\left[a_{1}, f\left(a_{1}\right)\right)$. Hence $M\left[a_{1}, a_{2}\right] \subset$ 
$M\left[a_{1}, f\left(a_{1}\right)\right)$ and $n>1$. Note that $a_{n+1} \notin M\left[a_{1}, f\left(a_{1}\right)\right]$ and $f\left(a_{1}\right) \notin$ $M\left[a_{1}, a_{n+1}\right]$. By (5), $a_{n+1} \in M\left[a_{1}, f\left(a_{n}\right)\right)$. Since $M$ does not contain a simple closed curve, it follows that $M\left[a, f\left(a_{n}\right)\right] \cap M\left[a, f\left(a_{1}\right)\right]=\{a\}$.

Suppose (7) is false. Then $a \notin f\left[M\left[a_{1}, a_{n}\right]\right]$. Consequently

$$
M\left[a, f\left(a_{1}\right)\right] \cup M\left[a, f\left(a_{n}\right)\right] \cup f\left[M\left[a_{1}, a_{n}\right]\right]
$$

contains a simple closed curve, and this violates the unique arcwise connectivity of $M$. Hence (7) is true.

For each point $c$ of $P(a)$,

(8) $c \in f\left[M\left[a_{1}, c\right)\right]$.

To establish (8) assume the contrary. Let $y$ be the point of $P(a)$ that is the greatest lower bound of $\left\{z \in P(a): z \notin f\left[M\left[a_{1}, z\right)\right]\right\}$ relative to $\prec$. By (7) and the continuity of $f$, there exists a point $x$ of $M\left[a_{1}, y\right)$ such that $f(x)=y$. Assume without loss of generality that $y \notin f[M(x, y)]$.

Suppose $x \in M\left[a_{i}, a_{i+1}\right)$ and $y \in M\left[a_{n}, a_{n+1}\right)$. By (1), (2), and (3), $n>i$ +1 . Since $M$ does not contain a simple closed curve, $M\left[y, a_{n+1}\right] \cap$ $f\left[M\left[x, a_{i+1}\right]\right]=\{y\}$. By (5), $a_{n+1} \in M\left[a_{1}, f\left(a_{n}\right)\right)$. Therefore $M\left[y, f\left(a_{n}\right)\right] \cap$ $M\left[y, f\left(a_{i+1}\right)\right]=\{y\}$. Since $y \notin f[M(x, y)]$, it follows that

$$
M\left[y, f\left(a_{n}\right)\right] \cup M\left[y, f\left(a_{i+1}\right)\right] \cup f\left[M\left[a_{i+1}, a_{n}\right]\right]
$$

contains a simple closed curve, and this violates the unique arcwise connectivity of $M$. Hence (8) is true.

For each integer $i>1$,

(9) there exists a positive integer $n$ such that $P\left(a_{n}\right) \cap f\left[M\left[a_{1}, a_{i}\right]\right]=\varnothing$.

To see this assume there exists an integer $i>1$ such that for each positive integer $n, P\left(a_{n}\right) \cap f\left[M\left[a_{1}, a_{i}\right]\right] \neq \varnothing$. Since $M$ does not contain a simple closed curve, there exists a positive integer $j$ such that $P\left(a_{j}\right) \subset f\left[M\left[a_{1}, a_{i}\right]\right]$. Since $f\left[M\left[a_{1}, a_{i}\right]\right]$ is a dendrite, $\operatorname{Cl} P\left(a_{j}\right)$ is a dendrite [11, Theorem 4, p. 301], and this contradicts (2) (see Theorem 5 of [11, p. 302]). Therefore (9) is true.

Let $L=\cap_{n=1}^{\infty} \mathrm{Cl} P\left(a_{n}\right)$. It follows from (2) that $L$ is not degenerate. Hence $L$ is a continuum.

According to Lemma 2, there exist a complementary domain $D$ of $L$ and a positive integer $\alpha$ such that $P\left(a_{\alpha}\right) \subset \mathrm{Cl} D$. Assume without loss of generality that $P \subset \mathrm{Cl} D, a_{1} \in D$ (Lemma 3 ), and $D$ is the unbounded complementary domain of $L$.

Let $X$ be the continuum $R^{2} \backslash D$. Since $M$ is arcwise connected, there is an arc segment $S$ in $M \cap D$ with an endpoint in $X$. Since $M$ does not contain a simple closed curve, we can assume without loss of generality that $P \cap \mathrm{Cl} S$ $=\varnothing$.

Using Sieklucki's nested sequence of polygonal disks (described in \$2 above), define a sequence $\left\{\mathscr{F}_{m}\right\}_{m=1}^{\infty}$ with the property that for each $m, \mathscr{F}_{m}$ is an $m$-chain on $(X, S)$ refined by $\mathscr{F}_{m+1}$. 
For each positive integer $m$, let $\mathcal{S}_{m}$ be the set of all elements $F$ of $\mathscr{F}_{m}$ such that for each point $u$ of $P, P(u)$ intersects (Int $F) \backslash X$. By Lemma 6, for each $m, \mathscr{G}_{m}$ is a subchain of $\mathscr{F}_{m}$. Note that if $m$ and $n$ are integers and $0<m<n$, then $\mathcal{G}_{n}$ refines $\mathcal{G}_{m}$ and each end link of $\mathcal{G}_{m}$ contains an end link of $\mathcal{S}_{n}$.

For each positive integer $m$, let $G_{1}^{m}, G_{2}^{m}, \ldots, G_{\lambda_{m}^{m}}^{m}$ be the consecutive links of $\mathcal{G}_{m}$.

By Lemma 7, for each positive integer $m$, there exists a point $u_{m}$ of $P \cap$ Int $G_{1}^{m}$ such that $P\left(u_{m}\right)$ is a trace of $\mathcal{G}_{m}$. Hence for each $m, L \subset \mathrm{St} \mathcal{G}_{m}$. Since $M$ does not contain a simple closed curve, it follows from the proof of Lemma 6 that for each $m$, $L$.

(10) no arc segment in $(M \backslash X) \cap \operatorname{St}\left\{G_{i}^{m}: 1<i<\lambda_{m}\right\}$ has an endpoint in

For each positive integer $m$, there exists an arc $B_{m}$ in $P\left(u_{m}\right)$ such that $f\left[B_{m}\right] \subset\left(\right.$ St $\left.\mathcal{G}_{m}\right) \backslash X$ and $f\left[B_{m}\right]$ is a trace of $\mathcal{G}_{m}$. To see this let $v$ be a point of $P\left(u_{m}\right) \cap$ Int $G_{\lambda_{m}}^{m}$. By (8), (9), and (10), there exist points $w$ and $x$ of $P(v)$ such that $f(w) \in P(w) \cap$ Int $G_{1}^{m}, f(x) \in P(x) \cap$ Int $G_{\lambda_{m}}^{m}$, and the arc $M[f(w), f(x)]$ is between $L$ and $M\left[u_{m}, v\right]$ in St $\mathcal{G}_{m}$. By (6), $M\left[u_{m}, v\right] \cap$ $f[M[w, x]]=\varnothing$. Since $M$ does not contain a simple closed curve, it follows from (10) that there exists a subarc $B_{m}$ of $M[w, x]$ such that $f\left[B_{m}\right] \subset\left(\mathrm{St}_{m}\right)$ $\backslash X$ and $f\left[B_{m}\right]$ is a trace of $\mathcal{G}_{m}$.

Note that $X$ has the following property:

Reduction Property. The continuum $X$ does not separate $R^{2}$ and there exist an arc segment $S$ in $M \backslash X$ with an endpoint in $X$, a sequence $\left\{A_{m}\right\}_{m=1}^{\infty}$ of arcs in $P$ converging to $B d X$, and a sequence $\left\{\mathcal{G}_{m}\right\}_{m=1}^{\infty}$ of chains such that for each $m$,

(i) $\mathcal{G}_{m}$ is a subchain of an $m$-chain on $(X, S)$,

(ii) $\mathcal{G}_{m+1}$ refines $\mathcal{G}_{m}$,

(iii) each end link of $\mathcal{G}_{m}$ contains an end link of $\mathcal{G}_{m+1}$,

(iv) $A_{m}$ agrees with $\mathcal{G}_{m}$, and

(v) either $f\left[A_{m}\right] \subset\left(\right.$ St $\left.\mathcal{G}_{m}\right) \backslash X$ or there exists a subarc $B_{m}$ of $A_{m}$ such that $f\left[B_{m}\right] \subset\left(\right.$ St $\left.\mathcal{G}_{m}\right) \backslash X$ and $f\left[B_{m}\right]$ agrees with $\mathcal{G}_{m}$.

Next we prove that $X$ contains a continuum that is irreducible with respect to the Reduction Property. Assume $\left\{X_{n}\right\}_{n=1}^{\infty}$ is a nested sequence of nonseparating plane continua in $X$. For each $n$, assume there exist an arc segment $S_{n}$ in $M \backslash X_{n}$ that has an endpoint in $X_{n}$, a sequence $\left\{A_{m}^{n}\right\}_{m=1}^{\infty}$ of $\operatorname{arcs}$ in $P$ converging to $\mathrm{Bd} X_{n}$, and a sequence $\left\{\mathcal{G}_{m}^{n}\right\}_{m=1}^{\infty}$ of chains with the following property. For each $m, \mathcal{G}_{m}^{n}$ is a subchain of an $m$-chain on $\left(X_{n}, S_{n}\right), \mathcal{G}_{m+1}^{n}$ refines $\mathcal{G}_{m}^{n}$, each end link of $\mathcal{G}_{m}^{n}$ contains an end link of $\mathcal{G}_{m+1}^{n}, A_{m}^{n}$ agrees with $\mathcal{G}_{m}^{n}$, and either $f\left[A_{m}^{n}\right] \subset\left(\right.$ St $\left.\mathcal{G}_{m}^{n}\right) \backslash X_{n}$ or there exists a subarc $B_{m}^{n}$ of $A_{m}^{n}$ such that $f\left[B_{m}^{n}\right] \subset\left(\right.$ St $\left.\mathcal{G}_{m}^{n}\right) \backslash X_{n}$ and $f\left[B_{m}^{n}\right]$ agrees with $\mathcal{G}_{m}^{n}$. Let $X_{0}=\bigcap_{n=1}^{\infty} X_{n}$. According to the Brouwer reduction theorem [16, p. 17], it is sufficient to prove that $X_{0}$ is a continuum with the Reduction Property. 
Since $f$ is continuous, for each positive integer $n$, either $f\left[\operatorname{Bd} X_{n}\right] \subset \operatorname{Bd} X_{n}$ or $\mathrm{Bd} X_{n} \subset f\left[\mathrm{Bd} X_{n}\right]$. Since $\left\{\mathrm{Bd} X_{n}\right\}_{n=1}^{\infty}$ converges to $\mathrm{Bd} X_{0}$, it follows that $f\left[\mathrm{Bd} X_{0}\right] \subset \operatorname{Bd} X_{0}$ or $\operatorname{Bd} X_{0} \subset f\left[\mathrm{Bd} X_{0}\right]$. Since $f$ moves each point of $M$, $\operatorname{Bd} X_{0}$ is not degenerate. Hence $X_{0}$ is a continuum.

Since $R^{2} \backslash X_{0}=\cup_{n=1}^{\infty} R^{2} \backslash X_{n}$ and for each $n, R^{2} \backslash X_{n}$ is connected, it follows that

(11) $R^{2} \backslash X_{0}$ is connected.

Since $M$ is arcwise connected, there is an arc segment $S_{0}$ in $M \backslash X_{0}$ with an endpoint in $X_{0}$. Assume without loss of generality that $P \cap \mathrm{Cl} S_{0}=\varnothing$.

Define a sequence $\left\{\mathscr{F}_{m}^{0}\right\}_{m=1}^{\infty}$ with the property that for each $m, \mathscr{F}_{m}^{0}$ is an $m$-chain on $\left(X_{0}, S_{0}\right)$ refined by $\mathfrak{F}_{m+1}^{0}$.

There exists a sequence $\left\{\mathcal{G}_{m}^{0}\right\}_{m=1}^{\infty}$ of chains such that for each $m$,

(12) $\mathcal{G}_{m}^{0}$ is a subchain of $\mathscr{F}_{m}^{0}$,

(13) $\mathcal{G}_{m+1}^{0}$ refines $\mathcal{G}_{m}^{0}$,

(14) each end link of $\mathcal{G}_{m}^{0}$ contains an end link of $\mathcal{G}_{m+1}^{0}$, and

(15) there exist integers $i_{m}$ and $j_{m}\left(j_{m}>m\right)$ such that

(i) $\mathrm{St} \mathcal{G}_{i_{m}}^{j_{m}} \subset\left(\mathrm{St} \mathcal{G}_{m}^{0}\right) \backslash \mathrm{Bd}\left(X_{0} \cup \mathrm{St} \mathscr{F}_{m}^{0}\right)$,

(ii) the interior of each interior link of $\mathcal{S}_{m}^{0}$ contains the sides of two consecutive links of $\mathcal{G}_{i_{m}}^{j_{m}}$,

(iii) no endpoint of a side of a link of $\mathcal{G}_{m}^{0}$ belongs to $A_{i_{m}}^{j_{m}} \cup f\left[A_{i_{m}}^{j_{m}}\right]$ (recall (6)), and $m^{-1}$.

(iv) the Hausdorff distance [11, p. 47] from $A_{i_{m}}^{j_{m}}$ to $\mathrm{Bd} X_{j_{m}}$ is less than

For each positive integer $m$, let $A_{m}^{0}=A_{i_{m}}^{j_{m}}$. Since $\left\{\operatorname{Bd} X_{n}\right\}_{n=1}^{\infty}$ converges to Bd $X_{0}$, it follows from (15(iv)) that

(16) $\left\{A_{m}^{0}\right\}_{m=1}^{\infty}$ converges to Bd $X_{0}$.

By (15(i)-(iii)) and Lemma 5, for each positive integer $m$,

(17) $A_{m}^{0}$ agrees with $\mathcal{S}_{m}^{0}$, and either

(18) $f\left[A_{m}^{0}\right] \subset\left(\right.$ St $\left.\mathcal{S}_{m}^{0}\right) \backslash X_{0}$, or

(19) there exists a subarc $B_{m}^{0}$ of $A_{m}^{0}\left(B_{m}^{0}=B_{i_{m}}^{j_{m}}\right)$ such that $f\left[B_{m}^{0}\right] \subset\left(\operatorname{St} \mathcal{G}_{m}^{0}\right) \backslash$ $X_{0}$ and $f\left[B_{m}^{0}\right]$ agrees with $\mathcal{S}_{m}^{0}$.

It follows from (11)-(14) and (16)-(19) that $X_{0}$ has the Reduction Property. Hence there exists a subcontinuum of $X$ that is irreducible with respect to the Reduction Property.

For convenience we assume that

(20) no proper subcontinuum of $X_{0}$ has the Reduction Property.

According to Lemma 4, there exists a positive integer $\beta$ such that $\varepsilon>2^{1-\beta}$ and no pair of consecutive links of $\mathcal{G}_{\beta}^{0}$ contains Bd $X_{0}$ in its union.

By (6), $S_{0} \cap f[P(u)]=\varnothing$ for some point $u$ of $P$. Assume without loss of generality that for each integer $m \geqslant \beta$,

(21) $S_{0} \cap f\left[A_{m}^{0}\right]=\varnothing$. 
Let $G_{1}, G_{2}, \ldots, G_{\gamma}$ be the consecutive links of $\mathcal{G}_{\beta}^{0}$. For $i=1,2, \ldots, \gamma-$ 1, let $V_{i}=\cup_{j=1}^{i} G_{j}$, and let $W_{i}$ be the common side of $G_{i}$ and $G_{i+1}$.

Let W $=\left\{W_{i}: 2 \leqslant i \leqslant \gamma-2\right\}$. Note that each element of of has diameter less than $\varepsilon$.

For $m=\beta, \beta+1, \ldots$ and $i=2,3, \ldots, \gamma-2$, let $W_{i}^{m}=A_{m}^{0} \cap W_{i}$.

Definition. A point $x$ of $W_{i}^{m}$ is sent back by $f$ if $f(x) \in V_{i}$; otherwise, $x$ is sent forward by $f$.

Definition. The arc $A_{m}^{0}$ has the switch property if a component of $A_{m}^{0} \backslash$ St $\mathcal{W}$ has endpoints in St $\mathcal{W}$ that are sent in opposite directions by $f$.

Statement (18) is true for only finitely many integers $m \geqslant \beta$. To see this assume the contrary. Suppose without loss of generality that (18) is true for each integer $m \geqslant \beta$.

For each integer $m \geqslant \beta$, if $f$ sends two points of $\cup_{i=2}^{\gamma-2} W_{i}^{m}$ in opposite directions, then, by (1), (18), and (21), $A_{m}^{0}$ has the switch property.

Suppose that for infinitely many integers $m \geqslant \beta$, two points of $\cup_{i=2}^{\gamma-2} W_{i}^{m}$ are sent in opposite directions by $f$. Then infinitely many elements of $\left\{A_{m}^{0}\right\}_{m=\beta}^{\infty}$ have the switch property. Assume without loss of generality that there exists a component $G$ of $\left(\mathrm{St}_{\beta}\right) \backslash\left(S_{0} \cup X_{0} \cup \mathrm{St} \mathscr{W}\right)$ such that for each integer $m \geqslant \beta, A_{m}^{0}$ has the switch property on a component $T_{m}$ of $A_{m}^{0} \backslash \mathrm{St}$ ข that is contained in $\mathrm{Cl} \mathrm{G}$.

For each integer $m \geqslant \beta$, we have three cases.

Case 1. Suppose $f\left[\mathrm{Cl} T_{m}\right] \subset G$. Then $\mathrm{Cl} G$ is a link of $\mathcal{G}_{m}^{0}$ and $T_{m}$ has an endpoint in each side of $\mathrm{Cl} G$.

Case 2. Suppose $f\left[\mathrm{Cl} T_{m}\right]$ intersects two components of $\left(\mathrm{St} \mathcal{G}_{\beta}^{0}\right) \backslash\left(G \cup S_{0}\right.$ $\left.\cup X_{0}\right)$. Then $\mathrm{Cl} G$ is a link of $\mathcal{G}_{\beta}^{0}$ and there exists an $\operatorname{arc} A$ in $\mathrm{Cl} T_{m}$ such that $f[A] \subset \mathrm{Cl} G$ and $f[A]$ intersects each side of $\mathrm{Cl} G$.

Case 3. Suppose $f\left[\mathrm{Cl} T_{m}\right]$ intersects only one component of $\left(\mathrm{St}_{\beta}^{0}\right) \backslash(G \cup$ $S_{0} \cup X_{0}$ ). Then there exist an element $W_{i}$ of $\mathscr{C}$ in $\mathrm{Cl} G$ and an $\operatorname{arc} A$ in $\mathrm{Cl} T_{m}$ with an endpoint in $W_{i}$ such that $f[A] \subset \mathrm{Cl} G$ and $W_{i} \cap f[A] \neq \varnothing$.

Since one of these three cases holds for infinitely many elements of $\left\{T_{m}\right\}_{m=\beta}^{\infty}$, there is a continuum $Y$ in $X_{0} \cap \mathrm{Cl} G$ with the following properties. A sequence $\left\{H_{m}\right\}_{m=1}^{\infty}$ of arcs in $P$ converging to $Y$ and a sequence $\left\{\mathcal{H}_{m}\right\}_{m=1}^{\infty}$ of chains exist such that for each positive integer $m$,

(22) $\mathrm{St} \mathcal{H}_{m} \subset \mathrm{Cl} \mathrm{G}$,

(23) $\mathcal{H}_{m}$ is a subchain of $\mathcal{G}_{m+\beta}^{0}$,

(24) $\mathcal{H}_{m+1}$ refines $\mathcal{H}_{m}$,

(25) each end link of $\mathcal{H}_{m}$ contains an end link of $\mathcal{H}_{m+1}$,

(26) $H_{m}$ agrees with $\mathcal{H}_{m}$, and

(27) either $f\left[H_{m}\right] \subset\left(\mathrm{St} \mathcal{H}_{m}\right) \backslash X_{0}$ or there exists a subarc $I_{m}$ of $H_{m}$ such that $f\left[I_{m}\right] \subset\left(\right.$ St $\left.\mathcal{H}_{m}\right) \backslash X_{0}$ and $f\left[I_{m}\right]$ agrees with $\mathcal{H}_{m}$.

Let $U$ be the complementary domain of $Y$ that contains $D$. Let $X^{\prime}$ be the nonseparating plane continuum $R^{2} \backslash U$. Note that $Y=\operatorname{Bd} X^{\prime}$. 
Since $M$ is arcwise connected, there exists an arc segment $S^{\prime}$ in $M \backslash X^{\prime}$ with an endpoint in $X^{\prime}$. Since $M$ does not contain a simple closed curve, we can assume without loss of generality that $P \cap \mathrm{Cl} S^{\prime}=\varnothing$.

Define a sequence $\left\{g_{m}\right\}_{m=1}^{\infty}$ with the property that for each $m, g_{m}$ is an $m$-chain on $\left(X^{\prime}, S^{\prime}\right)$ refined by $g_{m+1}$.

There exists a sequence $\left\{\mathcal{K}_{m}\right\}_{m=1}^{\infty}$ of chains such that for each $m$,

(28) $\mathcal{K}_{m}$ is a subchain of $\mathcal{G}_{m}$,

(29) $\mathcal{K}_{m+1}$ refines $\mathcal{K}_{m}$,

(30) each end link of $\mathcal{K}_{m}$ contains an end link of $\mathcal{K}_{m+1}$, and

(31) there exists an integer $i_{m}$ such that

(i) St $\mathcal{H}_{i_{m}} \subset\left(\right.$ St $\left.\mathcal{K}_{m}\right) \backslash \operatorname{Bd}\left(X^{\prime} \cup \operatorname{St} \mathscr{g}_{m}\right)$,

(ii) the interior of each interior link of $\mathcal{K}_{m}$ contains the sides of two consecutive links of $\mathcal{H}_{i_{m}}$, and

(iii) no endpoint of a side of a link of $\mathcal{K}_{m}$ belongs to $H_{i_{m}} \cup f\left[H_{i_{m}}\right]$.

It follows from (26)-(31) that $X^{\prime}$ has the Reduction Property. Since $\mathrm{Cl} G$ is either a link or the union of two consecutive links of $\mathcal{G}_{\beta}^{0}, \mathrm{Bd} X_{0} \not \subset \mathrm{Bd} X^{\prime}$. But $X^{\prime}$ and $X_{0}$ are nonseparating plane continua and $\operatorname{Bd} X^{\prime} \subset \operatorname{Bd} X_{0}$. Consequently $X^{\prime}$ is a proper subcontinuum of $X_{0}$, and this contradicts (20). Hence for all but finitely many integers $m \geqslant \beta, f$ sends each point of $\cup_{i=2}^{\gamma-2} W_{i}^{m}$ in the same direction.

Assume without loss of generality that for each integer $m \geqslant \beta$, every point of $\cup \underset{i=2}{\gamma-2} W_{i}^{m}$ is sent back by $f$.

The set $\left\{m: f\left[A_{m}^{0} \cap\left(G_{1} \cup G_{2}\right)\right] \not \subset G_{1} \cup G_{2}\right\}$ is finite; for otherwise, Case 3 (with $\mathrm{Cl} G=G_{1} \cup G_{2}$ and $W_{i}=W_{2}$ ) holds for infinitely many elements of $\left\{A_{m}^{0}\right\}_{m=\beta}^{\infty}$, and we have shown that this is impossible. Hence we can assume without loss of generality that for each integer $m \geqslant \beta$,

(32) $f\left[A_{m}^{0} \cap\left(G_{1} \cup G_{2}\right)\right] \subset G_{1} \cup G_{2}$.

For each positive integer $m$, let $\mathcal{H}_{m}$ be the chain consisting of all links of $\mathcal{G}_{m+\beta}^{0}$ that intersect $\operatorname{Int}\left(G_{1} \cup G_{2}\right)$, and let $H_{m}$ be an arc in $A_{m+\beta}^{0} \cap\left(G_{1} \cup G_{2}\right)$ that intersects $W_{2}$ and agrees with $\mathcal{H}_{m}$.

The sequence $\left\{H_{m}\right\}_{m=1}^{\infty}$ converges to a continuum in $X_{0} \cap\left(G_{1} \cup G_{2}\right)$. For each positive integer $m$, St $\mathcal{H}_{m} \subset G_{1} \cup G_{2}$ and conditions (23)-(26) are satisfied. By (18) and (32), for each positive integer $m, f\left[H_{m}\right] \subset\left(\mathrm{St} \mathcal{H}_{m}\right) \backslash$ $X_{0}$. According to the argument following (27), a proper subcontinuum of $X_{0}$ has the Reduction Property, and this contradicts (20). Hence (18) is true for at most finitely many integers.

Assume without loss of generality that (19) holds for each integer $m \geqslant \beta$.

By the preceding argument, for infinitely many integers $m \geqslant \beta, A_{m}^{0}$ does not have the switch property on a component of $A_{m}^{0} \backslash \mathrm{St}$ ข that is contained in $B_{m}^{0}$. Hence we can assume without loss of generality that for each integer $m \geqslant \beta$, every point of $B_{m}^{0} \cap \cup_{i=2}^{\gamma-2} W_{i}^{m}$ is sent forward by $f$. It follows from a similar argument that for infinitely many integers $m \geqslant \beta$, 
(33) for each point $x$ of $B_{m}^{0} \backslash V_{i}(2 \leqslant i \leqslant \gamma-2), f(x) \notin V_{i}$.

We assume without loss of generality that (33) holds for each integer $m \geqslant \beta$.

Since for each positive integer $m, B_{m+\beta}^{0}$ has the properties given in (19) and (33), there exist a sequence $\left\{H_{m}\right\}_{m=1}^{\infty}$ of arcs in $P$ converging to a continuum in $X_{0} \cap\left(G_{1} \cup G_{2}\right)$ and a sequence $\left\{\mathcal{H}_{m}\right\}_{m=1}^{\infty}$ of chains with the following properties. For each positive integer $m$, St $\mathcal{H}_{m} \subset G_{1} \cup G_{2}$, conditions (23)-(26) are satisfied, $H_{m} \cap W_{2} \neq \varnothing$, and there exists a subarc $I_{m}$ of $H_{m}$ such that $f\left[I_{m}\right] \subset\left(\mathrm{St} \mathcal{H}_{m}\right) \backslash X_{0}$ and $f\left[I_{m}\right]$ agrees with $\mathcal{H}_{m}$.

By the argument following (27), a proper subcontinuum of $X_{0}$ has the Reduction Property, and this contradicts (20). Hence every continuous function of $M$ into $M$ has a fixed point.

\section{REFERENCES}

1. W. L. Ayres, Some generalizations of the Scherrer fixed-point theorem, Fund. Math. 16 (1930), 332-336.

2. H. Bell, On fixed point properties of plane continua, Trans. Amer. Math. Soc. 128 (1967), 539-548.

3. D. P. Bellamy, Composants of Hausdorff indecomposable continua; a mapping approach, Pacific J. Math. 47 (1973), 303-309.

4. R. H. Bing, The elusive fixed point property, Amer. Math. Monthly 76 (1969), 119-132.

5. K. Borsuk, $A$ theorem on fixed points, Bull. Acad. Polon. Sci. 2 (1954), 17-20.

6. C. L. Hagopian, A fixed point theorem for plane continua, Bull. Amer. Math. Soc. 77 (1971), 351-354.

7. __ Another fixed point theorem for plane continua, Proc. Amer. Math. Soc. 31 (1972), $627-628$.

8. $\lambda$ connectivity and mappings onto a chainable indecomposable continuum, Proc. Amer. Math. Soc. 45 (1974), 132-136.

9. F. B. Jones, Concerning non-aposyndetic continua, Amer. J. Math. 70 (1948), 403-413.

10. J. Krasinkiewicz, Concerning the accessibility of composants of indecomposable plane continua, Bull. Acad. Polon. Sci. 21 (1973), 621-628.

11. K. Kuratowski, Topology, Vol. 2, 3rd ed., Monografie Mat., Tom 21, PWN, Warsaw, 1961; English transl., Academic Press, New York; PWN, Warsaw, 1968.

12. L. Mohler, The fixed-point property for homeomorphisms of 1-arcwise connected continua, Proc. Amer. Math. Soc. 52 (1975), 451-456.

13. R. L. Moore, Foundations of point set theory, rev. ed., Amer. Math. Soc. Colloq. Publ., vol. 13, Amer. Math. Soc., Providence, R.I., 1962.

14. H. E. Schlais, Non-aposyndesis and non-hereditary decomposability, Pacific J. Math. 45 (1973), 643-652.

15. K. Sieklucki, On a class of plane acyclic continua with the fixed point property, Fund. Math. 63 (1968), 257-278.

16. G. T. Whyburn, Analytic topology, rev. ed., Amer. Math. Soc. Colloq. Publ., vol. 28, Amer. Math. Soc., Providence, R.I., 1963.

17. G. S. Young, Fixed-point theorems for arcwise connected continua, Proc. Amer. Math. Soc. 11 (1960), 880-884.

Department of Mathematics, California State University, Sacramento, California 95819 